Doris Germain Philippe Crine Guy Boileau David Y. Thomas

\section{ADRESSES}

D. Germain : stagiaire de recherche. $\mathrm{Ph}$ Crine : professeur titulaire. G. Boileau : professeur agrégé. Département de biochimie, université de Montréal, case postale 6128 succ. A Montréal, H3C 3J5, Canada.

D. Y. Thomas : chef de section, génie génétique. Institut de recherche en biotechnologie, CNRC Royalmount, Montréal, H4P 2R2, Canada.

\title{
Protéolyse différentielle des prohormones : identification de convertases
}

Le clonage du gène de levure $K E X 2$, codant pour une enzyme de maturation endoprotéolytique des précurseurs de la phéromone $\alpha$ et des toxines tueuses (killer) $\mathrm{K} 1$ et $\mathrm{K} 2$, a fourni le premier modèle d'une convertase capable de transformer un précurseur polyprotéique de neuropeptides et d'hormones en ses produits actifs. C'est en partie grâce aux informations structurales ainsi recueillies que furent par la suite clonés les ADNc codant pour PC1 et PC2, deux convertases probablement impliquées dans la maturation de neuropeptides et d'hormones polypeptidiques, notamment de la POMC, le précurseur de l'ACTH. Les clivages provoqués par PC1 et PC2 sont différents et aboutissent à des peptides structuralement et fonctionnellement différents, ce qui mime la maturation différentielle de la POMC dans les lobes antérieur et intermédiaire de l'hypophyse. Les convertases sont elles-mêmes synthétisées sous la forme de précurseurs devant subir une maturation protéolytique. La conversion des précurseurs polyhormonaux peut donc être la conséquence d'une cascade d'activations mettant en jeu de nombreuses enzymes encore inconnues.

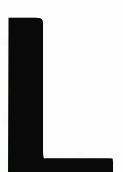

a plupart des hormones et des neuropeptides du système nerveux central sont produites sous forme de précurseurs. Les domaines nécessaires à l'activité physiologique de ces peptides sont généralement délimités par un résidu arginine ou par une paire d'acides aminés basiques (Arg, Lys), suggérant qu'une enzyme de type trypsine intervient dans la libération de pep- tides actifs. Cette hypothèse a pour la première fois, été proposée, pour la conversion de la pro-insuline en insuline [1] et pour la conversion de la $\beta$-lipotropine en $\beta$-mélanotropine [2]. Le modèle de protéolyse spécifique fut par la suite généralisé à plusieurs autres hormones peptidiques et neurotransmetteurs [3]. En 1977, la découverte du précurseur de l'ACTH et de la $\beta$-endorphine, appelé proopiomélanocortine (POMC) [4] permit 
1. Steiner DF, Cunningham D, Spiegelman $\mathrm{L}$, et al. Insulin biosynthesis : evidence for a precursor. Science $1967 ; 157$ : 697.

2. Chrétien $\mathrm{M}, \mathrm{Li} \mathrm{CH}$. Isolation and characterization of $\gamma$-lipotropic hormone from sheep pituitary glands. Can J Biochem 1967 ; $45: 1163-74$.

3. Douglass J, Civelli O, Herbert E. Polyprotein gene expression: generation of diversity of neuroendocrine peptides. Ann Rev Biochem 1984; 53 : 665-715.

4. Crine P, Gianoulakis C, Seidah NG, et al. Biosynthesis of $\beta$-lipotropin and a larger molecular weight precursors in rat pars intermedia. Proc Natl Acad Sci USA 1978; 75 : 4719-23.

5. Gomez S, Boileau G, Zollinger L, et al. Site specific mutagenesis identifies amino acid residues critical in prohormone processing. EMBO J $1989 ; 8$ : 1911-6.

6. Brakch $W$, Boussetta $H$, Rholam $M$, Cohen P. Processing endoprotease recognizes a structural feature at the cleavage site of peptide prohormones. J Bio Chem 1989 ; $264: 15912-6$

7. Rholam M, Nicolas $P$, Cohen P. Precursors for peptide hormones share common secondary structures forming features at the proteolytic processing sites. FEBS Lett 1986 ; $207: 1-6$.

8. Clamagirand C, Creminon C, Fahy C, et al. Partial purification and functional properties of an endoprotease from bovine neurosecretory granules cleaving proocytocin/neurophysin peptides at the basic amino acid doublet. Biochem $1987 ; 26$ : 6018-23.

9. Gomez S, Gluschankof P, Lepage A, Cohen P. Relationship between endo- and exopeptidases in a processing enzyme system : activation of an endoprotease by the aminopeptidase B-like activity in somatostatin-28 convertase. Proc Natl Acad Sci USA 1988 ; 85 : 5468-72.

10. Loh YP. Kinetics studies on the processing of human $\beta$-lipotropin by bovine pituitary intermediate lobe proopiomelanocortin-converting enzyme. J Biol Chem 1986; 261 : 11949-55.

11. Krieger TJ, Hook VYH. Purification and characterization of a novel thiol endoprotease involved in processing the enkephalin precursor. J Biol Chem 1991; 266 :
12. Davidson HHW, Rhodes CJ, Hutton JC. Intracellular organellar calcium and $\mathrm{pH}$ control proinsulin cleavage in the pancreatic $\beta$ cell via two distinct site-specific endopeptidases. Nature 1988 ; 333 : 93-6.

13. Julius D, Brake A, Blair L, Kunisawa $\mathrm{R}$, Thorner $\mathrm{J}$. Isolation of the putative structural gene for the lysine-argininecleaving endoprotease required for the processing of the yeast prepro- $\alpha$-factor. Cell $1984 ; 37: 1075-89$.

14. Bathurst IC, Brennan SO, Carrell RW, Cousens L, Brake AJ, Barr PJ. Yeast KEX2 protease has the properties of a human proalbumin converting enzyme. Science 1987 ; 235 : 348-50.

15. Thomas G, Thorne BA, Thomas L, et al. Yeast KEX2 endoprotease correctly cleaves a neuroendocrine prohormone in mammalian cells. Science $1988 ; 241: 226-30$.

16. Zollinger L, Racine C, Crine $\mathrm{P}$, et al. Intracellular proteolytic processing of proopiomelanocortin in heterologous COS-1 cells by yeast Kex2 endoprotease. Biochem Cell Biol 1990 ; 68 : 635-40.

17. Germain D, Zollinger L, Racine C, $e$ al. The yeast KEX2-processing endoprotease is active in the Golgi apparatus of transfected NIH 3T3 fibroblasts. Mol Endo 1990 ; $4: 1572-9$

18. Foster DC, Holly RD, Sprecher CA, Walker KM, Kumar AA. Endoproteolytic processing of the human protein $\mathrm{C}$ precursor by the Kex2 endoprotease coexpressed in mammalian cells. Biochemistry 1991; 30 : $367-72$.

19. Bresnahan PA, Leduc $R$, Thomas L, et al. Human fur gene encodes a yeast KEX2-like endoprotease that cleaves pro- $\beta$ NGF in vivo. J Cell Biol $1990 ; 111$ : 2851-9.

20. Van de Van WJM, Voorberg J, Fontijn $\mathrm{R}$, et al. Furin is a subtilisin-like proprotein processing enzyme in higher eucaryotes. Mol Biol Rep 1990 ; 14 : 265-75.

21. Hatsuzawa K, Hosaka M, Nakagawa $\mathrm{T}$, et al. Structure and expression of mouse furin, a yeast Kex2-related protease. J Biol Chem 1990 ; 265 : 22075-8.

22. Smeekens SP, Steiner DF. Identification of a human insulinoma cDNA encoding a novel mammalian protein structurally related to the yeast dibasic processing protease Kex2. J Biol Chem 1990; 265 : 2997-3000. d'étendre ce concept à des précurseurs contenant plusieurs peptides, doués chacun d'une activité biologique différente. Ces précurseurs multiples, ou polyprotéines, permettent de diversifier, au niveau posttraductionnel, les peptides actifs engendrés à partir d'un seul précurseur.

La complexité du processus de protéolyse différentielle implique la reconnaissance sélective de certains sites. Bien que certaines caractéristiques structurales des régions adjacentes à ces sites semblent importantes pour diriger les protéases de maturation [5-6], il n'existe aucun consensus quant au rôle des séquences primaires [7]. De plus, les sites possibles de protéolyse d'un même précurseur, tel que la POMC, sont reconnus différemment selon les tissus où le précurseur est exprimé. Ces observations suggèrent l'existence de plusieurs endoprotéases de maturation.

Pendant plusieurs années, les chercheurs ont tenté d'isoler par les méthodes classiques de la biochimie ces enzymes de maturation communément appelées « convertases ". Bien que certains candidats aient été partiellement isolés et caractérisés [8-12], ce n'est que récemment que les $\mathrm{ADN}$ c de certains membres de cette famille d'enzymes ont pu être clonés et leur structure primaire déterminée. Ces travaux constituent un jalon important pour l'étude des mécanismes qui contrôlent l'expression des hormones polypeptidiques et des neuropeptides. La caractérisation de ces convertases devrait permettre l'élucidation de mécanismes fondamentaux inhérents à la biochimie du système nerveux central. De plus, tout progrès dans ce sens pourrait avoir des retombées cliniques puisque certaines de ces enzymes pourraient être des candidates pour la maturation de protéines telles que la substance $\beta$ amyloïde, associée à la maladie d'Alhzeimer, ainsi que de certains facteurs de croissance.

\section{Identification \\ d'une protéase de maturation chez la levure}

Une étape importante dans l'identification des convertases des systèmes 


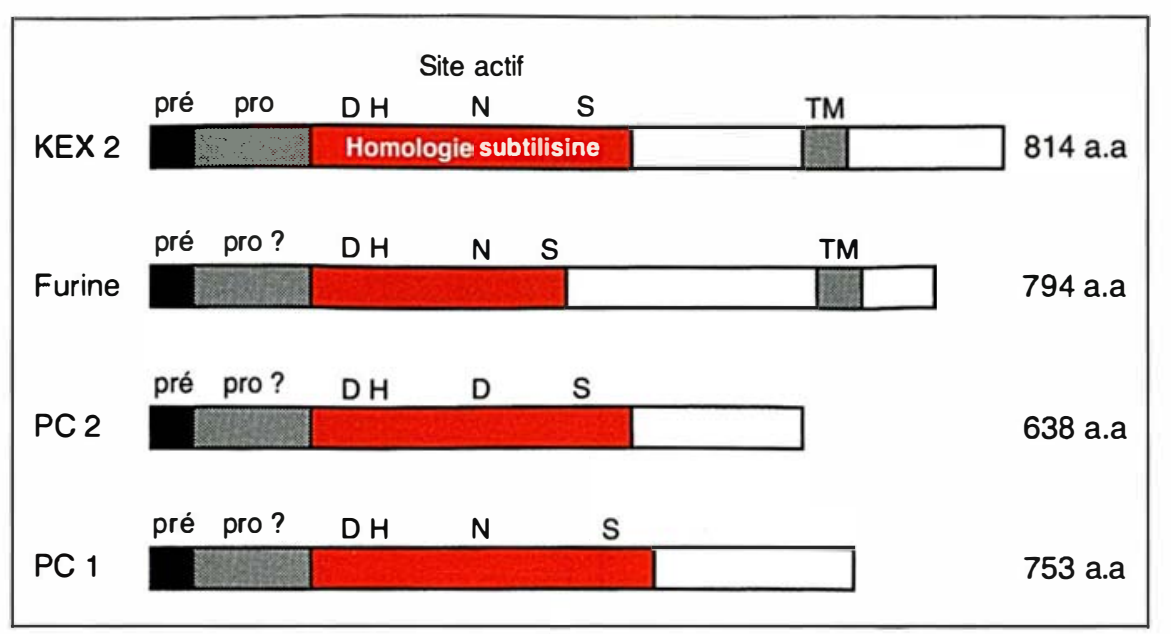

Figure 1. Structures des convertases du type-Kex2. Les différents domaines sont représentés : peptide signal (Pré), Pro-peptide (Pro), site actif, région trans-membranaire (TM). La présence de séquences Pro dans la furine, PC1 et PC2 n'a pas encore été démontrée. Les acides aminés impliqués dans la fonction catalytique sont également représentés dans leur code à une lettre. $D:$ Asp ; $H=$ His : $N$ : Asn; $S:$ Ser.

neuroendocriniens des mammiferes fut l'identification d'une protéase de maturation chez un eucaryote unicellulaire : Saccharomyces cerevisiae (plus connu sous le nom de levure de bière). Cette levure peut exister sous trois types cellulaires: les formes haploïdes MAT $a$ et MAT $\alpha$, et la forme diploïde MAT $a /$ MAT $\alpha$. Le passage de la forme haploïde à la forme diploïde se fait par conjugaison sexuelle entre cellules haploïdes de types différents et nécessite la sécrétion de peptides actifs dénommés facteurs $a$ et $\alpha$. Le type haploïde MAT $\alpha$ produit le facteur $\alpha$ sous forme de précurseur contenant quatre copies du facteur $\alpha$ mûr encadrées par des paires d'acides aminés basiques.

En 1984, le groupe de Jeremy Thorner a caractérisé une souche mutante de $S$. cerevisiae incapable de produire, à la fois, le facteur $\alpha$ mûr et une seconde protéine, issue elle aussi d'un précurseur, la toxine "Killer 2 ". Le gène responsable fut cloné et nommé $K E X 2$ [13]. La protéine, codée par le gène $K E X 2$, possède un domaine d'homologie avec une protéase à sérine, la subtilisine (figure 1). De plus, la protéine Kex2 possède une activité endoprotéolytique spécifique des sites dibasiques. Cette enzyme semble donc posséder certaines caractéristiques d'une convertase. Néanmoins, l'importance de la protéine Kex2 en $m / s n^{\circ} 9$, vol. 7 , novembre 91 teur de croissance pro- $\beta$ NGF [19] et du précurseur du facteur von Willebrand [20]. La maturation de ces protéines s'effectue au niveau de sites dibasiques. En revanche, la furine de souris ne semble pas capable d'induire la maturation de la pro-rénine [21] et, à ce jour, aucune maturation de prohormone par la furine n'a été rapportée. De plus, la furine semble être présente dans tous les tissus, même ceux où l'on n'observe pas l'expression de prohormones. Cette distribution ubiquitaire ne peut expliquer la spécificité de la protéolyse à l'intérieur de certains tissus, notamment le cerveau, et laisse donc présager la présence d'autres enzymes agissant indépendamment ou de concert avec la furine. Les chercheurs eurent donc recours à la technologie de l'amplification d'ADN par PCR pour procéder à l'identification d'autres enzymes de la même famille. Le groupe de Steiner a utilisé des oligonucléotides dérivés des séquences conservées entourant le site actif de la protéine Kex2 et de la subtilisine pour amplifier les ADNc d'une banque d'insulinome humain. Ce travail permit l'identification d'un ADNc contenant une seule phase de lecture de $1914 \mathrm{pb}$, désigné hPC2 [22]. Au même moment, un second groupe, celui de Seidah et Chrétien, utilisait des oligonucléotides dérivés cette fois des séquences conservées entre la furine et Kex2, pour amplifier les ADNc d'une banque d'hypophyse de souris. Leur travail permit l'identification d'un premier clone, ayant une phase de lecture ouverte de $2250 \mathrm{pb}$, désigné $\mathrm{mPC} 1$, et d'un second clone, de $1911 \mathrm{pb}$, désigné mPC2 [23].

Finalement, un troisième $\mathrm{ADNc}$, désigné $\mathrm{PC} 3$, fut isolé des cellules AtT20, une lignée cellulaire tumorale d'hypophyse de souris [24]. Ce dernier correspond à mPC1. Il semble donc y avoir, à ce jour, trois convertases potentielles de type Kex2 chez les mammiferes : la furine, PC1 et $\mathrm{PC} 2$. ainsi que la furine pourrait être une convertase. Afin de vérifier cette hypothèse, on exprima le gène de la furine par transfection dans des cellules de mammifères. On put démontrer que la furine humaine peut entraîner la maturation in vivo du fac-

\section{Localisation et spécificité de PC1 et PC2 chez la souris}

Contrairement à la furine, l'expres- 
Peptide N-terminal
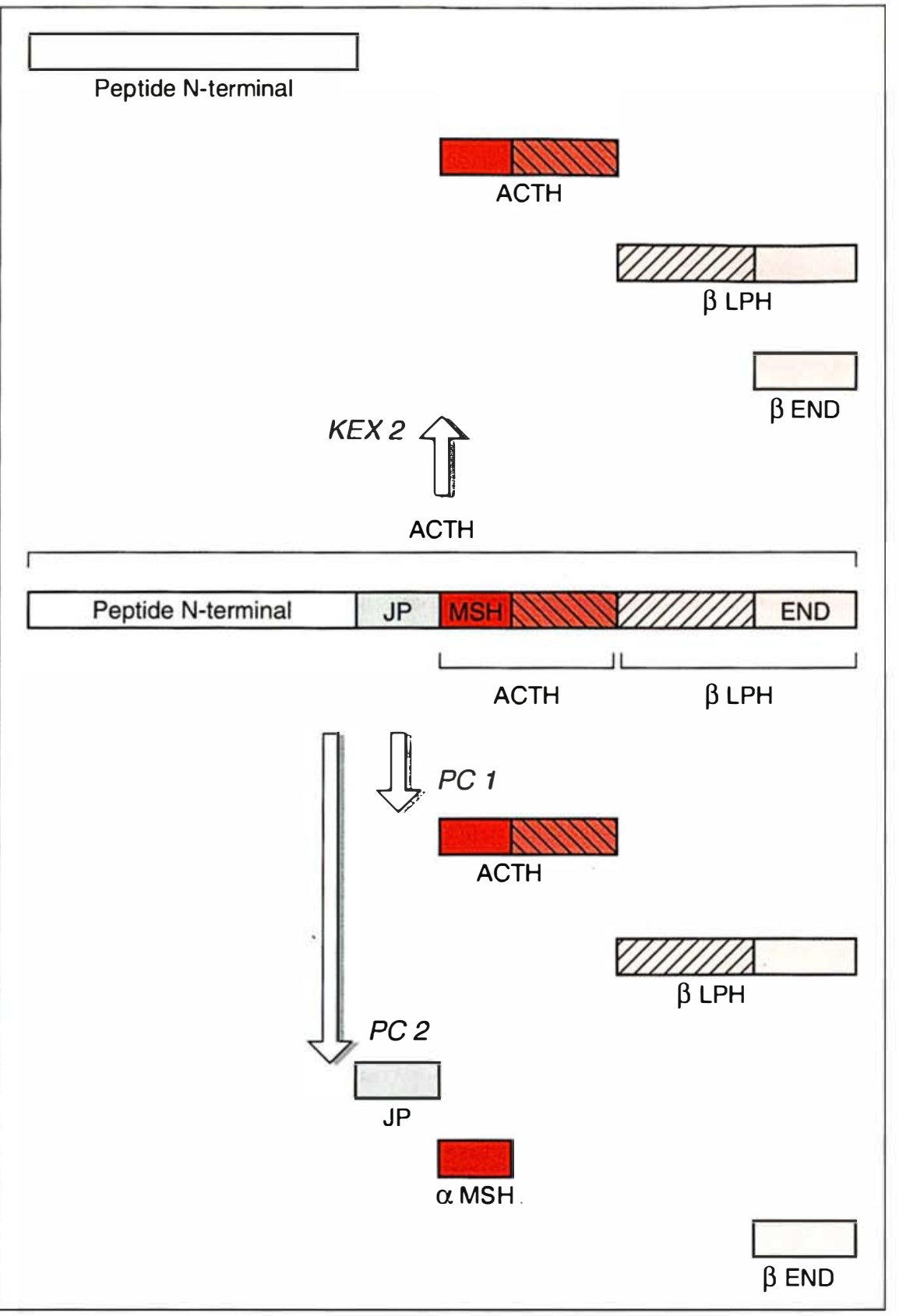

\section{RÉFÉRENCES}

23. Sildah NG, Gaspar L, Mion P, Marcinkiewicz $\mathbf{M}$, Mbikay $\mathbf{M}$, Chrétien $\mathbf{M}$. cDNA sequence of two distinct pituitary proteins homologous to Kex2 and furin gene products : tissue-specific mRNAs encoding candidates for the prohormone processing proteinases. DNA Cell Biol 1990; 9 : 415-24.

24. Smeekens SP, Avruch AS, LaMendola J, Chan SJ, Steiner DF. Identification of a cDNA encoding a second putative prohormone convertase related to PC2 in AtT20 cells and islets of Langerhans. Proc Natl Acad Sci USA 1991; 88: 340-4.

25. Seidah NG, Marcinkiewicz M, Benjannet $\mathrm{S}$, et al. Cloning and primary sequence of a mouse candidate prohormone convertase PC1 homologous to PC2, furin, and Kex2 : distinct chromosomal localization and messenger RNA distribution in brain and pituitary compared to PC2. Mol Endo 1990 ; 5 : 111-22.

26. Benjannet $\mathrm{S}$, Rondeau $\mathrm{N}$, Day $\mathrm{R}$, Chrétien M, Seidah NG. PC1 and PC2 are proprotein convertases capable of cleaving proopiomelanocortin at distinct pairs of basic residues. Proc Natl Acad Sci USA 1991 ; $88: 3564-8$.

27. Mackin RB, Noe BD. Direct evidence for two distinct prosomatostatin converting enzymes. J Biol Chem 1987 ; 262 : 6453-6.

28. Beinfeild $M$, Bourdais $J$, Kuks $P$, Morel A, Cohen P. Characterization of an endoprotease from rat small intestinal mucosal secretory granules which generates somatostatin- 28 from prosomatostatin by cleavage after a single arginine residue. $J$ Biol Chem 1989; 264 : 4460-5.

29. Bourbonnais Y, Danoff A, Thomas DY, Shields D. Heterologous expression of peptide hormone precursors in the yeast Saccharomyces cerevisiae: evidence for a novel prohormone endoprotease with specificity for monobasic amino acids. J Biol Chem 1991; in press.

30. Coll M, Guasch A, Aviles FX, Huber $\mathrm{R}$. Three dimensional structure of porcine procarboxypeptidase B : a structural basis

Figure 2. Protéolyse différencielle du précurseur de la POMC par les convertases Kex2, PC1 et PC2. Seuls les produits de maturation de la POMC identifiés à ce jour sont représentés. JP : joining peptide (peptide de jonction). ACTH : adrénocorticotropine. $\beta$-LPH : $\beta$-lipoprotéine. $\beta E N D: \beta$-endorphine. $\alpha M S H$ : $\alpha$-mélanotropine.

sion de PC1 et PC2 est limitée à certains tissus tels que le cerveau, le pancréas et l'hypophyse. Le foie, le rein, le cœur et les intestins n'expriment par ces deux ARNm à un niveau détectable [24]. Une étude approfondie de localisation par hybridation in situ sur différentes sections du cerveau a permis de cartographier l'expression de PC1 et PC2 [25].
Cette étude démontre notamment que PC1 est exprimé dans les lobes antérieur et intermédiaire de la glande pituitaire, alors que PC2 est exprimé uniquement dans le lobe intermédiaire. Cette observation soulève la possibilité intéressante que l'expression conjointe ou individuelle de PC1 et PC2 est responsable de la maturation différentielle de précur- 
seurs tels que la POMC dans les lobes antérieur et intermédiaire. Cette hypothèse est renforcée par les résultats d'analyses de spécificité de coupure de la POMC par PC1 et PC2. En effet, PC1 coupe la POMC à deux des sites dibasiques, libérant ainsi l'adrénocorticotropine $(\mathrm{ACTH})$ et la $\beta$-lipotropine $(\beta$-LPH), alors que PC2 découpe la POMC à cinq sites dibasiques, libérant l' $\alpha$ mélanotropine $(\alpha-\mathrm{MSH})$ et la $\beta$ endorphine ( $\beta$-END) [26] (figure 2).

\section{Conclusion}

\section{et perspectives futures}

Les deux dernières années ont été le témoin d'une explosion des connaissances dans le domaine des convertases. Ce succès repose principalement sur l'exploitation d'un principe de conservation des séquences au cours de l'évolution. Malgré la forte similarité de séquences observée entre la subtilisine, Kex2, la furine, PC1 et PC2, une différence surprenante existe au cœur même des sites actifs de ces convertases. Des études du mécanisme catalytique des subtilisines ont démontré que non seulement la triade Asp, His et Ser est nécessaire à l'activité enzymatique, mais qu'en plus un résidu Asn situé à proximité de cette triade est également important pour la fonction catalytique des subtilisines. Kex2, la furine et PC1 possèdent un tel résidu Asn. En revanche, ce résidu est remplacé par un résidu Asp dans la séquence de $\mathrm{PC} 2$. Cette substitution suggère qu'un environnement acide est nécessaire à l'activité optimale de PC2 [24]. Selon cette hypothèse, l'activité de PC2 pourrait être restreinte aux organites acides tels que les granules de sécrétion.

Une autre différence importante entre ces convertases est que Kex2 et la furine sont toutes deux des enzymes transmembranaires, alors que $\mathrm{PC} 1$ et $\mathrm{PC} 2$ ne possèdent pas de séquences d'ancrage aux membranes (figure 1). Ces deux dernières fonctionnent néanmoins à l'intérieur des cellules, ce qui a amené certains chercheurs à suggérer que la présence d'une hélice amphipatique puisse être responsable de la rétention intracellulaire de PC1 et PC2 [25]. De plus, la présence de deux ARNm distincts $\mathrm{m} / \mathrm{s} n^{\circ} 9$, vol. 7 , novembre 9 pour PC1 et PC2 pourrait suggérer l'existence de deux isoformes, une forme soluble et une autre transmembranaire [22]. La vérification de cette hypothèse devra attendre la purification et la caractérisation de ces protéines.

Finalement, la présence de sites dibasiques, notamment dans la région $\mathrm{N}$ terminale de ces convertases, suggère qu'elles-mêmes sont produites sous formes de précurseurs. La détermination de la séquence $\mathrm{N}$-terminale de la protéine Kex2 démontre, effectivement, une maturation au site dibasique $\mathrm{Lys}_{107}-\mathrm{Arg}_{108}$ (résultats non publiés). Cette maturation est dépendante de l'activité de Kex2, ce qui suggère donc une automaturation de la forme proKex2 en Kex2. A ce jour, trois fonctions ont été associées aux pro-peptides : (1) la synthèse d'enzymes sous forme de zymogènes inactifs; (2) la facilitation de la sécrétion; et, finalement (3) le repliement correct de la protéine. $\mathrm{La}$ détermination du rôle de la prorégion de ces convertases représente un domaine de recherche prometteur puisque le développement d'inhibiteurs capables de constituer de nouveaux agents pharmacologiques repose sur une meilleure compréhension des mécanismes d'activation de ce type de protéases.

Des progrès énormes ont été réalisés dans la caractérisation des convertases spécifiques des sites dibasiques. Cependant, il est important de mentionner que plusieurs précurseurs sont clivés en des sites monobasiques, ce qui suggère l'existence d'au moins une autre classe de convertases. D'ailleurs, certaines convertases monobasiques ont été détectées chez le poisson [27], les mammiferres [28] et la levure [29]. Il est possible que certaines convertases soient responsables de l'activation d'autres enzymes de maturation, telles que les carboxypeptidases [30]. La clé de la régulation fine de la protéolyse différentielle repose donc possiblement sur une cascade d'événements d'activation intra- et intermoléculaire entre enzymes de maturation. Quoi qu'il en soit, il apparaît de plus en plus évident que le mécanisme de protéolyse différentiel est complexe et pourrait faire intervenir d'autres protéases, dont certaines attendent leur jour de gloire depuis quelques années déjà dans plusieurs laboratoires de par le monde

\section{Summary}

Differential proteolysis of prohormones : identification of mammalian endoproteases

In mammals, most active peptide hormones or neuropeptides are rcleased from precursor proteins of higher molecular weight. The specific endoproteases involved in the processing of these precursors have been difficult to isolate and characterize biochemically, primarly due to their low concentrations in the cell. In the yeast Saccharomyces cerevisiae, maturation of the $\alpha$ factor pheromone precursor and of the $\mathrm{K} 1$ and $\mathrm{K} 2$ killer toxin precursors requires the endoprotease Kex2. Expression of this yeast endoprotease in mammalian cells reveals that Kex2 is a potential homologuc of the maturation enzymes in higher eukaryotes. Three enzymes have becn isolated from mammalian cells based on their sequence similarity with Kex2. These proteins are furin, PC1 and PC2. Studies of localization and substrate specificity of these proteins confirmed their role in processing of prohormones. However, the heterogencity of the processing sites in prohormones underlines the necessity for the intervention of many other maturation enzymes. Candidates for these other enzymes have been reported.

\section{TIRÉS A PART}

Ph. Crine 